\title{
Schlieren imaging of inverted domains in congruent lithium niobate
}

\author{
M. Paturzo, P. Ferraro, S. Grilli, P. De Natale \\ Istituto Nazionale di Ottica Applicata Via Campi Flegrei 34, 80078 Pozzuoli'(Na), Italy \\ S. De Nicola, A. Finizio \\ Istituto di Cibernetica "E. Caianiello" del CNR, Via Campi Flegrei 34, 80078 Pozzuoli (Na), Italy \\ S. Mailis, R. W. Eason \\ Optoelectronics Research Centre, University of Southampton, Highfield, Southampton, SOI7 IBJ, UK
}

\begin{abstract}
:
We report Schlieren imaging of inverted domains in congruent $\mathrm{LiNbO}_{3}$. High contrast imaging is possible by taking advantage of the residual refractive index discontinuity at domain boundaries which is present even after prolonged thermal annealing.
\end{abstract}




\title{
Schlieren imaging of inverted domains in congruent lithium niobate
}

\author{
M. Paturzo, P. Ferraro, S. Grilli, P. De Natale \\ Istituto Nazionale di Ottica Applicata Via Campi Flegrei 34, 80078 Pozzuoli (Na), Italy \\ S. De Nicola, A. Finizio \\ Istituto di Cibernetica "E. Caianiello" del CNR, Via Campi Flegrei 34, 80078 Pozzuoli (Na), Italy \\ S. Mailis, R. W. Eason \\ Optoelectronics Research Centre, University of Southampton, Highfield, Southampton, SOI7 IBJ, UK
}

Non destructive observation of domain inverted regions in lithium niobate is of great importance for the quality assessment of domain engineered or periodically poled nonlinear devices based on this material, especially in integrated optical arrangements where the surface quality requirement prevents the use of methods such as chemical etching.

Here we report the observation of significant optical contrast enhancement at the boundaries of inverted domain areas in congruent lithium niobate, without the application of an external electric field, by the use of collimated coherent illumination along the $\mathrm{z}$ axis of the lithium niobate sample in a Schlieren optical arrangement. The detection of the inverted domains is possible due to the residual refractive index difference which exists between the newly poled areas and the virgin crystal and which remains in the vicinity of the inverted domain boundary even after thermal annealing. This residual refractive index change is of order $\Delta \mathrm{n} \sim 10^{-4}-10^{-5}$ and is responsible for the parasitic "zero voltage" signal in electrooptic devices which use inverted ferroelectric domain-engineered structures [1].

Using digital holographic interferometry techniques [2] to evaluate the magnitude of this residual refractive index between inverted domains over various annealing steps we found that there is a residual background refractive index difference between the newly poled area and the virgin crystal which is of order $\Delta \mathrm{n} \sim 10^{-4}$ and which gradually decreases with subsequent thermal annealing. Optical Schlieren images corresponding to the progressive annealing steps show a gradual reduction of the inverted domain visibility due to the reduction of the refractive index step. Finally, after annealing at $500^{\circ} \mathrm{C}$ for four hours any remaining index difference between the newly poled areas and the virgin, non-inverted, domain region appears to be fully removed. However, the residual refractive index discontinuity on the domain boundary is sufficient to produce a phase contrast image with a Schlieren optical setup, and an optical image of such a domain-inverted area obtained with this optical arrangement is shown in figure 1. This sample has an arrangement of square domain-inverted areas with a width of $450 \mu \mathrm{m}$ and has been subjected to thermal annealing at $500^{\circ} \mathrm{C}$ for four hours. Finer domain inverted features have been examined using this method in order to show its utility in the assessment of periodically poled structures. Shown in figure 2 is a Schlieren optical image of a hexagonally poled structure, showing also some limited domain merging in the area near the centre. The period of the structure here is of order $\sim 30 \mu \mathrm{m}$.

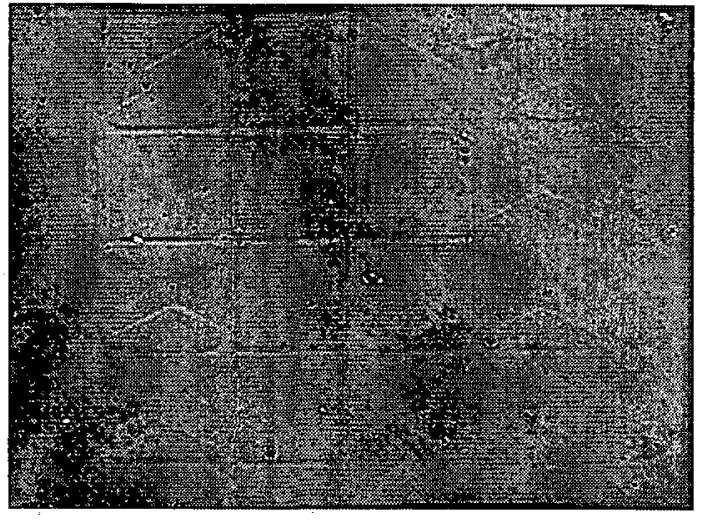

Figure 1. Imaging of inverted domain areas in lithium niobate crystal after four hours of thermal annealing at $500^{\circ} \mathrm{C}$ using a Schlieren optical arrangement.

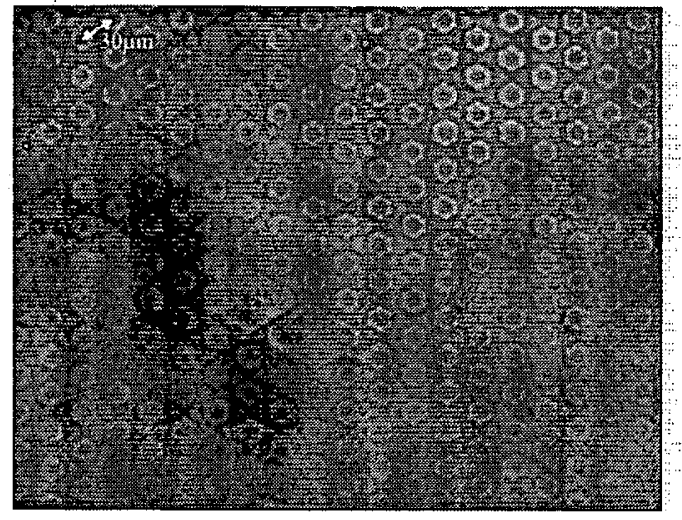

Figure 2. Schlieren image of a hexagonally poled lithium niobate structure. The period of the structure is $\sim 30 \mu \mathrm{m}$

1. A. J. Boyland, S. Mailis, J. M. Hendricks, P. G. R. Smith, R. W. Eason "Electro-optically controlled beam switching via total internal reflection at a domain-engineered interface in LiNbO " Opt. Comm.197 pp.193200(2001)

2. S. Grilli, P. Ferraro, S. De Nicola, A. Finizio, G. Pierattini, and R. Meucci, "Whole optical wave-fields reconstruction by digital holography," Opt. Express 9, 294-302 (2001) 